\title{
Genetic and environmental risk assessment for colorectal cancer risk in primary care practice settings: a pilot study
}

Ronald E. Myers, PhD ${ }^{1}$, David S. Weinberg, MD, MSc ${ }^{4}$, Sharon L. Manne, PhD ${ }^{5}$, Randa Sifri, $M D^{2}$, James Cocroft, $M A^{1}$, Kathryn Kash, $P h D^{3}$, and Benjamin Wilfond, $M D^{6}$

\begin{abstract}
Purpose: The assessment of genetic variants and environmental exposures (i.e., genetic and environmental risk assessment) may permit individualized risk stratification for common diseases as part of routine care. A pilot study was conducted to assess the uptake of, and response to, testing for colorectal cancer risk among average risk patients in primary care practice settings. Methods: Physicians in primary care practices identified patients eligible for colorectal cancer screening and referred them to the study. Research staff administered a baseline survey to consenting patients. At a scheduled office visit, participants underwent decision counseling with a trained nurse educator to facilitate informed decision making about being tested for methylene tetrahydrofolate reductase status and red blood cell folate level. Combined assessment can stratify colorectal cancer risk. Test results were disclosed within 2 weeks after the visit. Postvisit and 1-month endpoint surveys were administered. Univariable analyses of survey data were performed to assess changes from baseline in genetic and environmental risk assessment and colorectal cancer screening-related knowledge and perceptions. Results: Of the 57 patients who were referred to the study, 25 (44\%) consented to participate, and all but one were tested. Participant knowledge about genetic and environmental risk assessment and colorectal cancer screening, perceived colorectal cancer screening response efficacy, and perceived social support for colorectal cancer screening increased significantly from baseline. Participants reported low levels of intrusive thoughts about CRC. Conclusion: Knowledge and favorable perceptions of colorectal cancer screening increased, as did knowledge about genetic and environmental risk assessment, after exposure to the study intervention. Further research is needed to assess genetic and environmental risk assessment uptake and impact at the population level. Genet Med 2007:9(6):378-384.
\end{abstract}

Completion of the Human Genome Project has set the stage for the use of genetic information to personalize medical care. ${ }^{1}$ One important implication is that predictive genetic tests that attempt to stratify disease risk may be offered to asymptomatic individuals. This type of application may aid in determining who is at increased risk of common diseases such as diabetes, heart disease, and cancer. ${ }^{2}$

For most illnesses, the interaction between genetic and environmental factors, such as diet or physical activity, influences

From the Departments of ${ }^{1}$ Medical Oncology, ${ }^{2}$ Family and Community Medicine, and ${ }^{3}$ Psychiatry and Human Behavior, Jefferson Medical College, Thomas Jefferson University, Philadelphia, Pennsylvania; ${ }^{4}$ Gastroenterology Section, Fox Chase Cancer Center, Philadelphia, Pennsylvania; ${ }^{5}$ Division of Population Sciences, Fox Chase Cancer Center, Philadelphia, Pennsylvania; ${ }^{6}$ Treuman Katz Center for Pediatric Bioethics, Seattle Children's Hospital, Seattle, Washington.

Ronald E. Myers, PhD, Department of Medical Oncology, Jefferson Medical College, Thomas Jefferson University, 1025 Walnut Street, Suite 1014, College Building, Philadelphia, PA 19107; e-mail: ron.myers@mail.tju.edu

Disclosure: The authors declare no conflict of interest.

Submitted for publication January 12, 2007.

Accepted for publication March 19, 2007.

DOI: 10.1097/GIM.0b013e3180654ce2 risk. Combining genetic and environmental risk assessment (GERA) would enhance prognostic capabilities. In addition, information gained may help to target recommendations for risk reduction strategies for individual patients. However, the impact of GERA on clinical outcomes will depend on patient uptake of testing, understanding of test results and limitations, and adoption of recommended preventive behaviors. ${ }^{3}$

The predominant paradigm that has guided genetic risk assessment over the past two decades has involved the provision of extensive preparatory education and counseling related to genetics, documentation of informed consent, and disclosure of results by a trained genetic counselor. This approach was developed to inform high-risk individuals and their families about issues involved in detecting uncommon single gene disorders. This process requires a substantial amount of time and effort by highly trained professionals working in specialty settings. Testing for modestly predictive risk factors implicated in common diseases will require a different paradigm. To satisfy the much larger service requirements of average risk populations, GERA testing probably will be performed in primary care settings by existing staff. In preparation for this transition, it is necessary to gain insights into the feasibility of GERA in primary care. 
In the study described here, a trained nurse conducted a theory-based decision counseling session with participants related to having GERA for colorectal cancer (CRC) risk. CRC was selected as a model case for the current study because of emerging evidence that polymorphisms of the methylene tetrahydrofolate reductase (MTHFR) gene, an important regulator of cellular folate metabolism, and folate level (environment) can influence risk of CRC. Most results of published studies demonstrate an inverse relationship between folate intake or blood folate levels and CRC risk. ${ }^{4}$ In addition, the majority of reports suggest that joint assessment of common polymorphisms of MTHFR at nucleotide 677 and folate level can stratify CRC risk. ${ }^{5-7}$

It is reasonable to hypothesize that GERA results, which provide more personalized information about disease susceptibility, may influence CRC screening utilization. Novel methods to increase CRC screening rates in the general population are sorely needed. ${ }^{8}$ This pilot study was conducted to identify factors influencing participant decision making about having GERA and to evaluate changes in knowledge, perceived risk of CRC, perceived usefulness of screening, and distress among study participants.

\section{METHODS}

\section{Participant recruitment and enrollment}

Five primary care physicians (three general internists and two family physicians) prospectively identified potential participants from their patient populations. Adults between 50 and 74 years of age who had no personal history of CRC, inflammatory bowel disease, or any first-degree relative with CRC were eligible. All participants had to be asymptomatic for $\mathrm{CRC}$ and not currently compliant with standard CRC screening recommendations. This study was reviewed and approved by institutional review boards at Thomas Jefferson University, Fox Chase Cancer Center, and the National Human Genome Research Institute.

\section{Procedures}

A research nurse contacted all referred participants by telephone, confirmed eligibility, and administered a baseline survey questionnaire. Subsequently, interested participants were mailed a study consent and descriptive information about GERA and CRC screening (available from the investigators). Finally, arrangements were made for the study nurse and participants to meet after the next scheduled primary care visit.

Baseline survey data included age, gender, race, ethnicity, marital status, and level of education. Ten true-false items measured knowledge about GERA (framed in terms of diet and gene testing for CRC risk) were developed for this study using information from prestudy focus groups where laypersons, similar to study volunteers, participated in discussions regarding the GERA concept. Items used to measure knowledge about GERA are provided in the Appendix. Five true-false items measuring knowledge about CRC screening were also included on the survey. ${ }^{9}$
Perceptions related to GERA were assessed, based on the Preventive Health Model (PHM). The PHM is based on a selfregulation framework which posits that informed decision making involves the consideration of information about reasonable, available alternatives with the subsequent identification of a preferred alternative. ${ }^{10}$ Two items each, using Likerttype scales $(1=$ strongly disagree to $5=$ strongly agree $)$, were included to measure salience and coherence of having GERA, perceived likelihood of having an abnormal test result, worries and concerns about having GERA, GERA self-efficacy (i.e., belief that one can complete the assessment), GERA response efficacy (i.e., belief in the benefits of having the assessment), and GERA social support (i.e., belief in physician support for having the assessment). PHM scales used in previous studies regarding CRC screening were modified for use in relation to GERA. PHM scale reliability coefficients have been reported elsewhere. ${ }^{11,12}$ Interest in having GERA was measured using a single item ("I am interested in having colorectal cancer screening."). Perceptions related to CRC screening were measured along the same parameters.

A meeting after the primary care visit between the research nurse and study participant followed a scripted format. After written consent was collected, the research nurse and the participant reviewed the GERA and CRC screening brochures. In relation to GERA, the nurse highlighted the fact that persons with higher folate levels tend to be at lower risk of CRC. In addition, the nurse explained that certain types of MTHFR genes (polymorphisms) affect folate metabolism differently sometimes resulting in lower folate levels. The nurse educator described how persons 50 years and older with specific combinations of folate level and MTHFR polymorphism have a modestly increased risk of CRC compared with similar individuals without those combinations. Finally, the nurse educator highlighted the fact that increasing age is also an important risk factor for CRC and that all individuals 50 years and older should have CRC screening. The nurse educator reinforced the referring physician's support for patient use of one of the currently recommended CRC screening strategies (i.e., annual stool blood testing, flexible sigmoidoscopy every 5 years, annual stool blood testing and flexible sigmoidoscopy every 5 years, double contrast barium enema $\mathrm{x}$-ray every 5 years, or colonoscopy every 10 years). ${ }^{13}$

After reviewing the educational materials, the nurse educator led a decision counseling session regarding GERA. Each participant identified the top three "decision factors" that were likely to influence personal decision making about having GERA and to rank order these factors in terms of influence. Participants were guided through a process to assign weights to each decision factor using a seven-level visual analogue scale to indicate degree of influence on decision making (i.e., no influence to overwhelming influence). The same scale was employed to weight each decision factor relative to the others.

Decision factor weights were entered into a handheld computer containing software used to determine each participant's GERA preference score. Participant scores were categorized as low preference for GERA, moderate preference for GERA, or 
high preference for GERA. Details concerning this scoring system have been described elsewhere. ${ }^{10}$ For each participant, the nurse reviewed GERA preference, verified the accuracy of GERA preference, and elicited a GERA decision. For participants who decided to have GERA, separate consent was obtained and phlebotomy was performed for MTHFR genotyping and folate assessment.

At the end of the office visit, all study participants were given a postvisit survey to complete and return by mail. This survey repeated items from the baseline survey measuring knowledge about GERA and CRC screening and perceptions about CRC screening. In addition, seven items that form the Intrusion Subscale of the Impact of Events Scale-Revised were adapted for CRC and were included on the postvisit survey to assess whether participants had intrusive thoughts about CRC. ${ }^{14}$ One month later, study participants were mailed a third survey that measured perceptions about CRC screening and whether the participant reported intrusive thoughts about CRC.

\section{GERA testing, results reporting, and follow-up}

MTHFR polymorphism status and serum folate level, respectively, were assessed in laboratories located at Fox Chase Cancer Center and Thomas Jefferson University. Each laboratory sent test results to the study nurse, who then generated an overall report for the participant's primary care physician. Based on information displayed in Table 1, the report indicated CRC risk as "elevated" or "average" based on the MTHFR 677 genotype status and red blood cell folate level. We characterized GERA results in a manner consistent with the published data that attempted to avoid conveying false information about the precision of risk estimates. After receipt of the GERA report, the physicians contacted each participant by telephone to discuss the results. No record of GERA results was placed in the participant's office chart.

\section{Data analysis}

Patients with GERA knowledge, CRC screening knowledge, and perceptions about CRC screening scores available from baseline and postvisit surveys were included in the analysis. We also compared postvisit survey with endpoint survey measures of perceptions about CRC screening and the impact of intrusive thoughts about CRC. Differences in scores were compared using Wilcoxon signed-rank test. Nonparametric testing was used due to the skewness of the distribution of the scale measurements. Medians and ranges are reported as they reflect the differences compared by nonparametric tests. Two-sided tests are reported, and statistical significance is considered for $P<$ 0.05 .

\section{RESULTS}

Of the 57 eligible patients referred to study personnel, 25 (44\%) were successfully enrolled. Thirteen (23\%) potential participants could not be contacted by telephone. Of the 19 (33\%) who declined to participate, eight cited comorbid conditions, eight expressed low interest in joining a research study, and three were worried about abnormal GERA results. Study personnel were unsuccessful in obtaining a blood specimen from one patient who completed the GERA decision counseling session and consented to phlebotomy. This individual was excluded from relevant data analyses.

\section{Participant characteristics}

Table 2 displays demographic characteristics and baseline survey results for the 24 study participants from whom blood was collected. Study participants tended to be white (71\%), male (58\%), and married (72\%) with more than a high school education (88\%). Their mean age was 57 years. Almost two thirds of the participants provided correct answers to $50 \%$ or more of the survey items measuring GERA knowledge, whereas nearly $90 \%$ correctly answered the majority of CRC screening knowledge items. Not surprisingly, interest in and perceptions about GERA were positive in the group that had agreed to study enrollment. Interest in CRC screening was also high.

\section{Decision factors and preference related to GERA}

During decision counseling, participants identified a total of 65 decision factors ( 58 pro factors and seven con factors). Examples of pro factors included "I like to be informed about the status of my health," "My mother and some friends died of cancer, and I don't want that to happen to me," and "I owe it to my wife and children to be there for them." Examples of con factors were "No one in my family has colon problems," "I really don't like needles and blood draws," and "I don't want to know if I am at increased risk of cancer." Overall, more than $60 \%$ of the decision factors related to GERA identified by par-

Table 1

MTHFR 677 genotype, red blood cell folate level, risk category, and recommended response

\begin{tabular}{llll}
\hline Genotype & \multicolumn{1}{c}{ RBC folate } & Risk category & Recommended response \\
\hline TT & $\geq 145-540 \mathrm{ng} / \mathrm{mL}$ & Average risk & CRC screening \\
& $<145 \mathrm{ng} / \mathrm{mL}$ & Average risk & $\begin{array}{c}\text { CRC screening; physician and patient together should examine underlying cause (possibly use a folate } \\
\text { supplement }(400 \mu \mathrm{g} / \mathrm{d}) \text { and keep alcohol intake to } \leq 5 \text { drinks per week }\end{array}$ \\
CT/CC & $\geq 145-540 \mathrm{ng} / \mathrm{mL}$ & Average risk & $\begin{array}{c}\text { CRC screening } \\
\text { CRC screening; physician and patient together should examine underlying cause (possibly use a folate } \\
\text { supplement }(400 \mu \mathrm{g} / \mathrm{d}) \text { and keep alcohol intake to } \leq 5 \text { drinks per week }\end{array}$ \\
\hline
\end{tabular}

MTHFR, methylene tetrahydrofolate reductase; RBC, red blood cell; CRC, colorectal cancer. 
Table 2

Characteristics of study participants at baseline $(N=24)$

\begin{tabular}{|c|c|c|}
\hline Sociodemographic background & No. & $\%$ \\
\hline \multicolumn{3}{|l|}{ Age } \\
\hline $50-59$ y & 15 & 62 \\
\hline $60-74$ y & 9 & 38 \\
\hline \multicolumn{3}{|l|}{ Race } \\
\hline White & 17 & 71 \\
\hline African American & 7 & 29 \\
\hline \multicolumn{3}{|l|}{ Ethnicity } \\
\hline Non-Hispanic & 24 & 100 \\
\hline \multicolumn{3}{|l|}{ Gender } \\
\hline Male & 14 & 58 \\
\hline Female & 10 & 42 \\
\hline \multicolumn{3}{|l|}{ Education } \\
\hline High school graduate or less & 3 & 13 \\
\hline Some college & 6 & 25 \\
\hline College graduate & 15 & 62 \\
\hline \multicolumn{3}{|l|}{ Marital status } \\
\hline Married & 18 & 75 \\
\hline Not married & 6 & 25 \\
\hline \multicolumn{3}{|l|}{ GERA } \\
\hline \multicolumn{3}{|l|}{ Knowledge } \\
\hline$<50 \%$ correct & 9 & 38 \\
\hline$\geq 50 \%$ correct & 15 & 63 \\
\hline Perceptions & Median & Min, $\max$ \\
\hline Salience and coherence & 4.5 & $3.0,5.0$ \\
\hline Susceptibility & 2.0 & $1.0,4.0$ \\
\hline Worries and concerns & 2.0 & $1.0,4.0$ \\
\hline Self-efficacy & 4.0 & $2.0,5.0$ \\
\hline Response efficacy & 4.5 & $3.0,5.0$ \\
\hline Social support & 4.0 & $3.0,5.0$ \\
\hline Interest & 5.0 & $1.0,5.0$ \\
\hline Colon cancer screening & No. & $\%$ \\
\hline \multicolumn{3}{|l|}{ Knowledge } \\
\hline$<50 \%$ correct & 3 & 12 \\
\hline$\geq 50 \%$ correct & 21 & 88 \\
\hline Perceptions & Median & Min, Max \\
\hline Salience and coherence & 5.0 & $3.0,5.0$ \\
\hline Susceptibility & 3.0 & $1.0,4.0$ \\
\hline Worries and concerns & 2.3 & $1.0,4.0$ \\
\hline Self-efficacy & 4.5 & $2.0,5.0$ \\
\hline Response efficacy & 4.3 & $3.0,5.0$ \\
\hline Social support & 4.5 & $3.0,5.0$ \\
\hline Interest & 5.0 & $3.0,5.0$ \\
\hline
\end{tabular}

GERA, genetic and environmental risk assessment. ticipants were affective in nature. GERA preference scores were as follows: one participant had a low preference for GERA, five had a moderate preference for GERA, and 19 had a high preference for GERA.

\section{GERA acceptance and results}

As mentioned above, all 25 initial study enrollees agreed to provide blood for genotyping and folate assessment. Phlebotomy was unsuccessful in one instance. Only one participant carried the TT phenotype. None of the remaining participants with either the CC or CT genotype had a red blood cell folate value $<145 \mathrm{ng} / \mathrm{mL}$. Therefore, all participants were told that their risk of $\mathrm{CRC}$ was average.

\section{Changes in knowledge and perceptions among participants}

Table 3 displays data at baseline, postvisit, and endpoint survey data for the 18 study participants who completed all survey instruments. Changes in GERA knowledge and CRC screening knowledge were assessed by comparing correct responses to items included on the baseline survey and the postvisit survey. We observed that both GERA knowledge and CRC screening knowledge increased significantly $(P=0.0002$ and $P=0.0043$, respectively.)

Changes in CRC screening perceptions were assessed using data from the baseline, postvisit, and endpoint surveys. From completion of the baseline survey to the postvisit survey, we observed that CRC screening response efficacy, in other words, the perception that CRC screening was beneficial increased significantly $(P=0.002)$. From the time of postvisit survey completion to endpoint survey completion, perceived social support for CRC screening increased significantly $(P=0.035)$. We did not observe any other significant changes in CRC screening perceptions at these different time points. Finally, intrusive thoughts about CRC were infrequent at the time of the postvisit survey and at endpoint survey completion without any significant change over time.

\section{DISCUSSION}

In the future, testing and counseling services related to the assessment of personal risk of common diseases are likely to be offered as part of primary care. Formally trained genetic counselors are unlikely to participate routinely in the genetic testing process for frequent conditions seen in clinical practice. ${ }^{15-17}$ Competing time demands will force physicians and patients to approach decision making about genetic testing in new ways. ${ }^{18-20}$ This pilot study provides preliminary support for decision counseling by trained primary care nurses as a method to improve decision making by patients about genetic testing. In addition, it is one of the first to investigate the potential impact of GERA on patient knowledge and perception.

In this demonstration study, 57\% (25/44) of eligible participants who could be contacted in a timely fashion agreed to be in the study. Subsequently, all agreed to provide blood and to participate in PHM-based decision counseling. These findings are consistent with those of previous reports that a substantial 
Table 3

Comparison of baseline, postvisit, and endpoint measures $(N=18)$

\begin{tabular}{|c|c|c|c|c|c|c|c|c|c|}
\hline & \multicolumn{6}{|c|}{ Survey } & \multicolumn{3}{|c|}{$P^{a}$} \\
\hline & \multicolumn{2}{|c|}{ Baseline } & \multicolumn{2}{|c|}{ Postvisit } & \multicolumn{2}{|c|}{ Endpoint } & \multirow{2}{*}{$\begin{array}{c}\text { Baseline to } \\
\text { postvisit }\end{array}$} & \multirow{2}{*}{$\begin{array}{l}\text { Postvisit to } \\
\text { endpoint }\end{array}$} & \multirow{2}{*}{$\begin{array}{c}\text { Baseline to } \\
\text { endpoint }\end{array}$} \\
\hline & Median & Min, $\max$ & Median & Min, $\max$ & Median & Min, $\max$ & & & \\
\hline \multicolumn{10}{|l|}{ Knowledge $^{b}$} \\
\hline Diet and gene testing & 60 & 0,100 & 80 & 40,100 & - & - & 0.0002 & - & - \\
\hline Colon cancer and screening & 60 & 0,80 & 80 & 0,100 & - & - & 0.0043 & - & - \\
\hline \multicolumn{10}{|l|}{ CRC screening perceptions $^{c}$} \\
\hline Salience and coherence & 5.0 & 3,5 & 5.0 & 3,5 & 5.0 & 1,5 & 0.750 & 1.000 & 1.000 \\
\hline Susceptibility & 3.0 & 1,4 & 2.5 & $1.0,4.5$ & 2.0 & $1.0,4.5$ & 0.424 & 0.360 & 0.100 \\
\hline Worries and concerns & 2.2 & 1,4 & 3.0 & $1.0,4.5$ & 2.5 & 1,4 & 0.918 & 0.259 & 0.240 \\
\hline Self-efficacy & 4.5 & 2,5 & 5.0 & 1,5 & 4.5 & 1,5 & 0.185 & 0.836 & 0.356 \\
\hline Response efficacy & 4.3 & 3,5 & 4.7 & $3.3,5.0$ & 4.8 & $2.7,4.0$ & 0.002 & 0.908 & 0.092 \\
\hline Social support & 4.5 & 3,5 & 4.5 & $3.5,5.0$ & 5.0 & $3.5,5.0$ & 0.332 & 0.141 & 0.035 \\
\hline Interest & 5.0 & 3,5 & 5.0 & 2,5 & 5.0 & 3,5 & 0.375 & 0.375 & 1.000 \\
\hline IES-R intrusion subscale ${ }^{d}$ & - & - & 1.0 & $1.0,2.1$ & 1.0 & $1.0,1.7$ & - & 1.000 & - \\
\hline
\end{tabular}

CRC, colorectal cancer; IES-R, Impact of Events Scale-Revised.

${ }^{a}$ Wilcoxon signed-rank test.

${ }^{b}$ Ten true-false items for diet and gene testing knowledge; five true-false items for colon cancer and screening knowledge.

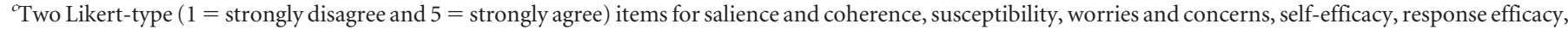
and social support. One item for interest.

${ }^{d}$ Seven items $(1=$ not at all, $2=$ sometimes, $3=$ rarely, $4=$ often $)$ to measure IES-R intrusion subscale.

proportion of the general population is interested in genetic testing for cancer susceptibility. ${ }^{21-24}$ However, the participants in this study who were open to the concept of genetic testing may not be representative of the broader group of primary care patients. The long-term value of genetic and environmental risk assessment will be limited unless there is general adoption by the public. Our study was not designed to gauge the overall degree of popular interest in GERA. One of the challenges for future research will be to encourage study enrollment for individuals who do not already have a high level of interest in GERA testing.

For those patients who did participate, exposure to decision counseling and GERA were associated with significant increases in knowledge about GERA and about CRC screening. Although GERA performance for CRC risk is novel, participants were able to grasp the basic concept of such testing. Further, we observed positive changes in CRC screening attitudes (i.e., greater perceived usefulness of CRC screening and increased perception of physician support for CRC screening) after decision counseling and GERA. Similar findings were reported in a study in which participants were educated about genetic risk of lung cancer and about smoking cessation. ${ }^{25,26}$ Here, these observed changes may have been due to information provided in the CRC screening brochure and by nurse educator encouragement of screening during the decision counseling session. Participants may have interpreted nurse educator support for screening as reflective of their physician's perspective related to screening.
Importantly, provision of information on CRC screening and GERA was not associated with intrusive thoughts about CRC. Observations about the safety and effectiveness of GERA, albeit in a small sample, provide important insights into the feasibility of such testing in primary care. Similar questions will need to be addressed in study populations in which some participants were informed of elevated risk based on GERA-like testing.

The clinical value of GERA or other approaches to geneticsbased personalized medicine will depend on its impact on health behaviors and outcomes. Larger studies must be carefully designed to distinguish the relationship between GERA participation and other factors that might influence screening behavior. For example, in this pilot study, the extent to which participants believed that their physician supported CRC screening increased significantly from baseline. Perceived provider support for screening is widely accepted as a positive influence on patient behavior. The perceived benefit of CRC screening also increased across the study time points. This is particularly noteworthy because none of the participants was deemed at elevated risk based on GERA results. Although GERA use may facilitate targeted screening efforts directed toward persons at elevated risk, it is also reasonable to hypothesize that GERA may increase overall screening participation through heightened attention to protective health behaviors.

This study has several limitations. The number of participants was small. We did not focus in this report on the impact of GERA on actual CRC screening use. Further, it should be 
noted that study participants were predominantly white and well educated. Our research group is currently conducting a randomized, controlled trial of GERA that is designed to include a substantially larger, heterogeneous population of individuals who are 50 years of age or older and eligible for CRC screening. In that study, we will assess population-based uptake of, participant attitudes about, and psychological effects of GERA. We will also use data collected in that investigation to perform more thorough analyses of psychological characteristics of study participants. These studies can also more closely examine GERA impact on folate intake as well as screening use. Studies designed to assess decision making related to GERA are also needed.

The growing literature on the effect of folate and MTHFR polymorphisms on CRC risk bespeaks a greater interest in the identification of clinically useful, biologically based risk stratification tools. At least for the short term, such tools will remain imprecise. It has been predicted that the next generation of genetic testing will be offered to healthy people who are, by standard criteria, at average risk of disease. This sort of risk assessment is already available to the public in unsupervised settings. It is presumed that commercial insurance will cover this sort of testing once its clinical utility is proven. Personalized molecular risk assessment for common diseases like cancer will not be useful unless the recipient incorporates the information as a valuable component in health care decision making. Future studies should consider not only how to optimize the quality of risk stratification tools, but also how best to use them in the context of routine health care. Decision counseling is one way to facilitate the informed use of effective tools.

\section{Appendix: Survey Items Used to Assess GERA Knowledge}

Please let us know what you think about each statement below. Check only one answer, true or false, for each statement.

1. My risk of colon cancer is not affected by dietary intake (that is, what I eat and drink).

2. My risk of colon cancer is affected by the types of genes I have.

3. My dietary intake and the types of genes I have work together to affect my risk of colon cancer.

4. A blood test can measure the different types of vitamins in my body.

5. A blood test cannot show the types of genes I have.

6. Diet and gene testing can show if I have colon cancer now.

7. Having a low level of folate in the body can protect me from colon cancer.

8. The types of genes I have do not affect my risk of colon cancer.

9. Results of a diet and gene test cannot show whether I should change the amount of folate I take in.
10. Diet and gene testing to determine colon cancer risk is commonly done in physician offices as part of routine care.

\section{ACKNOWLEDGMENTS}

Supported by National Institutes of Health 273-MH1122601 and Commonwealth of Pennsylvania, Department of Health ME01-329.

\section{References}

1. Collins FS. Shattuck lecture-medical and societal consequences of the Human Genome Project. N Engl J Med 1999;341:28-37.

2. Khoury MJ, Yang Q, Gwinn M, et al. An epidemiologic assessment of genomic profiling for measuring susceptibility to common diseases and targeting interventions. Genet Med 2004;6:38-47.

3. Marteau TM, Lerman C. Genetic risk and behavioural change. BMJ 2001; 322:1056-9.

4. Giovannucci E, Stampfer MJ, Colditz GA, Hunter DJ, et al. Multivitamin use, folate, and colon cancer in women in the Nurses' Health Study. Ann Intern Med 1998;129: 517-524.

5. Keku T, Millikan R, Worley K, Winkel S, et al. 5,10-Methylenetetrahydrofolate reductase codon 677 and 1298 polymorphisms and colon cancer in African Americans and whites. Cancer Epidemiol Biomarkers Prevent 2002;11:1611-1621.

6. Chen C, Ma J, Stampfer MJ, Palomeque C, et al. Linkage disequilibrium between the $677 \mathrm{C}>\mathrm{T}$ and $1298 \mathrm{~A}>\mathrm{C}$ polymorphisms in human methylenetetrahydrofolate reductase gene and their contributions to risk of colorectal cancer. Pharmacogenetics (2002);12:339-342.

7. Chen J, Giovannucci E, Hunter D. MTHFR polymorphism, methyl-replete diets and the risk of colorectal carcinoma and adenoma among U.S. men and women: An example of gene-environment interactions in colorectal tumorigenesis. J Nutr 1999; 129: 560S-564S.

8. Lieberman D. Screening for colorectal cancer in average-risk populations. Am JMed 2006;119:728-35.

9. Weinberg DS, Turner BJ, Wang H, Myers RE, et al. A survey of women regarding factors affecting colorectal cancer screening compliance. Prev Med 2004;38:669675.

10. Myers RE. Decision counseling in cancer prevention and control. Health Psychol 2005;24(Supplement4 suppl):S71-S77.

11. Vernon SW, Myers RE, Tilley BC. Development and validation of an instrument to measure factors related to colorectal cancer screening adherence. Cancer Epidemiol Biomarkers Prev 1997;6:825-832.

12. Tiro JA, Vernon SW, Hyslop T Myers RE. Factorial validity and invariance of a survey measuring psychosocial correlates of colorectal cancer screening among African Americans and Caucasians. Cancer Epidemiol Biomarkers Prev 2005;14:28552861.

13. Smith RA, Cokkinides V, Eyre HJ. American Cancer Society guidelines for the early detection of cancer, 2006. CA Cancer J Clin 2006;56:11-25.

14. Weiss D, Marmar C. The impact of event scale-revised. In: Wilson JP, Keane TM, editors. Assessing psychological trauma and PTSD. New York: Guilford Press; 1997: 577.

15. Wilfond BS, Fost N. The cystic fibrosis gene: medical and social implications for heterozygote detection. JAMA 1990;263:2777-2783.

16. Masny A, Daly M, Ross E, Balshem A, et al. A training course for oncology nurses in familial cancer risk assessment: evaluation of knowledge and practice. J Cancer Educ 2003; 18:20-25.

17. Wilson BJ. Improving the referral process for familial breast cancer genetic counselling: findings of three randomised controlled trials of two interventions. Health Technol Assess 2005;9:1-126.

18. Burke W. Genetic testing in primary care. Annu Rev Genomics Hum Genet 2004;5: $1-14$.

19. Wang C, Gonzalez R, Merajver SD. Assessment of genetic testing and related counseling services: current research and future directions. Soc Sci Med 2004;58:14271442 .

20. Burke W, Acheson L, Botkin J, Bridges K, et al. Genetics in primary care: a USA faculty development initiative. Community Genet 2002;5:138-146.

21. Smith KR, Croyle RT. Attitudes toward genetic testing for colon cancer risk. Am J Public Health 1995;85:1435-1438. 


\section{Myers et al.}

22. Andrykowski MA, Munn RK, Studts JL. Interest in learning of personal genetic risk for cancer: a general population survey. Prev Med 1996;25:527-536.

23. Graham ID, Logan DM, Hughes-Benzie R, et al. How interested is the public in genetic testing for colon cancer susceptibility? Report of a cross-sectional population survey. Cancer Prev Control 1998;2:167-172.

24. Satia JA, McRitchie S, Kupper LL, Halbert CH. Genetic testing for colon cancer among African-Americans in North Carolina. Prev Med 2006;42:51-59.
25. Lerman C, Gold K, Audrain J, Lin TH, et al. Incorporating biomarkers of exposure and genetic susceptibility into smoking cessation treatment: effects on smoking-related cognitions, emotions, and behavior change. Health Psycho 1997;16:87-99.

26. Audrain J, Boyd NR, Roth J, Main D, et al. Genetic susceptibility testing in smokingcessation treatment: one-year outcomes of a randomized trial. Addict Behav 1997; 22:741-751. 